\title{
Proteção de Sistema Fotovoltaico Conectado a Rede Elétrica
}

\author{
Protection of Grid Connected Photovoltaic System
}

\author{
Igor Vinicius Barros Gatis ${ }^{1}$ (D) orcid.org/0000-0002-3927-9518 \\ José Bione de Melo Filho ${ }^{1}$ (B) orcid.org/0000-0002-9283-3362 \\ ${ }^{1}$ Escola Politécnica de Pernambuco, Universidade de Pernambuco, Recife, Brasil, \\ E-mail do autor principal: Igor Gatis igorvbgatis@gmail.com
}

\section{Resumo}

O setor elétrico em termos de energia solar vem crescendo no Brasil, principalmente em relação a geração distribuída. Segundo a Resolução Normativa N482 da ANEEL pode-se dividir a geração distribuída em Microgeração e Minigeração. Já a partir da Resoluções Normativa 482 e a 687 vislumbra-se que o mercado de geração distribuída teve um crescimento expressivo, principalmente quanto aos sistemas fotovoltaicos. Entretanto, nota-se que a ausência de normas que regulamentam as instalações de corrente contínua de um sistema fotovoltaico está gerando erros durante o dimensionamento de proteções. Até que a NBR 16690 seja publicada, adota-se a NBR 5410, em que pode encontrar uma regulamentação de instalações de corrente contínua até $1500 \mathrm{~V}$. Há também a NBR5419 para regulamentar a necessidade de aterrar as partes condutoras expostas dos arranjos fotovoltaicos, para fins de proteção contra surtos provocados por descargas atmosféricas. Com esta visão do setor elétrico e tendo em vista o crescente número de instalações sendo realizadas, este artigo tem como objetivo informar e esclarecer as principais dúvidas quanto ao dimensionamento de suas proteções para as instalações em corrente contínua.

Palavras-Chave: proteção de sistemas fotovoltaicos; geração distribuída; sistemas de proteção para instalações fotovoltaicas

\begin{abstract}
The electric sector and the solar energy are currently growing, especially when it comes to distributed generation. According to the ANEEL Resolution 482, it is possible to separate the distributed generation into microgeneration and Minigeneration. Resolutions 482 and 687 make it clear that the distributed generation market has grown significantly (mainly in the photovoltaic systems). However, the wide range of regulations regarding the direct current installations of the Brazilian photovoltaic system is generating errors during the protection projects. Until NBR 16690 is published, NBR 5410 should be the regulation used, which contains DC installations of up to $1500 \mathrm{~V}$. There is also NBR5419, which regulates the need of grounding the exposed conductive parts of the photovoltaic array, in order to protect it against outbreaks caused by atmospheric discharges. With this vision of the electric sector and analyzing the growing number of installations being carried out, this article aims to inform and clarify the main doubts regarding the design of its protections for the installations in direct current.
\end{abstract}

Key-words: protection of photovoltaic systems; distributed generation; protection systems for photovoltaic installations 


\section{Introdução}

Diante da demanda exponencial no consumo de energia elétrica, verifica-se a crescente necessidade de um aumento em sua geração. Assim, tendo em vista a atuação dos órgãos ambientais e da mudança de pensamento da sociedade, em que passou a apresentar uma maior preocupação nas questões relacionadas à sustentabilidade, a busca por fontes de energias renováveis tem crescido vertiginosamente. Entre as formas de energia limpa e com poucos impactos ambientais, pode-se destacar o uso da energia solar, solução energética que satisfaz as atuais exigências da sociedade.

Um sistema solar pode ser projetado de grande a pequenas gerações (micro e minigeração), conectado à rede elétrica ou a um sistema isolado e com diversas aplicações possíveis, como bombeamento de água, iluminação, eletrificação rural, geração distribuída. Mesmo com essas aplicações, esse tipo de geração muitas vezes gera um custo elevado, o que dificulta sua utilização.

Sistemas Solares Fotovoltaicos ou simplesmente Sistemas Fotovoltaicos (FV), por sua vez, produzem tensões e correntes continuas elevadas, que geram riscos de choques elétricos. Desta forma, devem haver cuidados com instalação e manutenção contra descargas atmosféricas e outros fenômenos.

Uma vez que os sistemas fotovoltaicos ficam expostos ao meio ambiente, estão sujeitos a condições que podem gerar defeitos. Um aterramento, por exemplo, pode diminuir problemas, como os riscos de choques elétricos e de incêndios.

Sabe-se que o aterramento é a ligação das estruturas ou instalações com a terra, em que o objetivo é garantir seu funcionamento correto e proporcionar um caminho preferencial às correntes elétricas indesejáveis de surto, falta ou fuga, de forma a evitar riscos de choques elétricos para as pessoas e os equipamentos. Desta forma, este artigo vem abordar os principais pré-requisitos para executar aterramento do sistema FV em instalações de micro e minigeração distribuída.

Ao observar o aumento da geração distribuída em todo o Brasil, este trabalho tem como objetivo analisar e esclarecer os métodos de dimensionamento e instalação do sistema de proteção para usinas de geração distribuída. Tendo em vista que muitas dessas usinas são instaladas em residências, será abordado como a ligação do aterramento do circuito de corrente contínua deve ser interligado ao de corrente alternada em suas principais tipologias como TN-S, TN-C-S, TN-C, TT e IT, além de explicar como deve ser realizado o dimensionamento do dispositivo contra surto e dimensionamento de fusíveis ou disjuntores para corrente contínua.

\section{Aterramentos Elétricos}

Existem duas opções de aterramentos para sistemas fotovoltaicos, aterramento funcional (quando o arranjo fotovoltaico tem um condutor intencionalmente conectado à terra com o objetivo de garantir o correto funcionamento do sistema, ou seja, não tem propósito relacionado a segurança) e aterramento para proteção (ligação à terra de um ponto do arranjo fotovoltaico com propósito relacionado à segurança).

De acordo com a NBR 5410 (Instalações elétricas de baixa tensão), existem três esquemas de aterramento: TN (que possui 3 variantes), TT e IT [1].

Para caracterizá-los, utiliza-se os seguintes critérios:

A primeira letra identifica a situação da alimentação em relação a terra, em que o " $T$ " representa um ponto diretamente aterrado e a letra "I" representa uma isolação de todas as partes vivas em relação à terra ou aterramento de um ponto através de impedâncias[1].

A segunda, letra representa a situação das massas da instalação em relação a terra, em que o " $T$ " representa uma massa diretamente aterrada e a letra " $\mathrm{N}$ " representa massas ligadas ao ponto de alimentação aterrado[1].

Tem-se a existência de eventuais letras que representam variações do esquema de aterramento previamente adotado e representam a disposição do condutor neutro e do condutor de proteção, em que o $S$ representa as funções de neutro e de proteção asseguradas por condutores distintos e a letra " $\mathrm{C}$ " funções de neutro e de proteção combinadas em um único condutor[1].

\subsection{Esquema TN}

Neste tipo de esquema, possui um ponto e alimentação diretamente aterrado e as massas ligadas ao mesmo, através de condutores de proteção. Existem três variantes paras para o esquema TN, suas variações ocorrem por causa da disposição do 
condutor neutro e condutor de proteção, desta forma podendo variar de três maneiras diferentes:

Esquema TN-S: possui distintos condutores de neutro e de proteção. Esquema representado na figura $1[1]$.

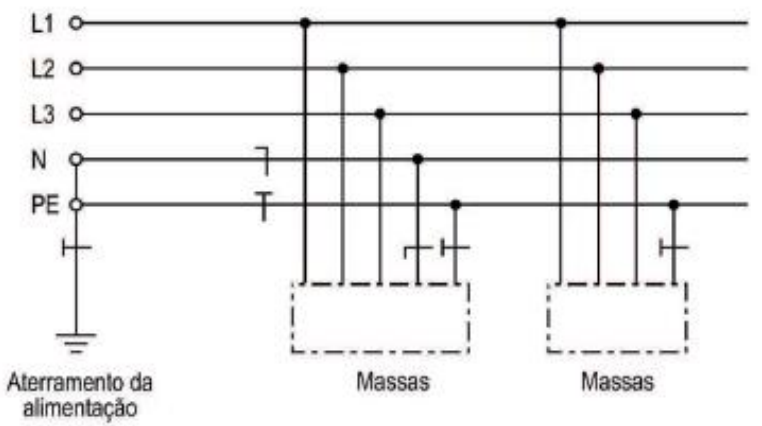

Figura 1: Representação do esquema TN-S.

Fonte: ABNT NBR 5410 (2004, p.15)

Esquema TN-C: possui um único condutor com as funções de neutro e proteção combinadas, em sua totalidade do esquema. Esquema representado na figura $2[1]$.

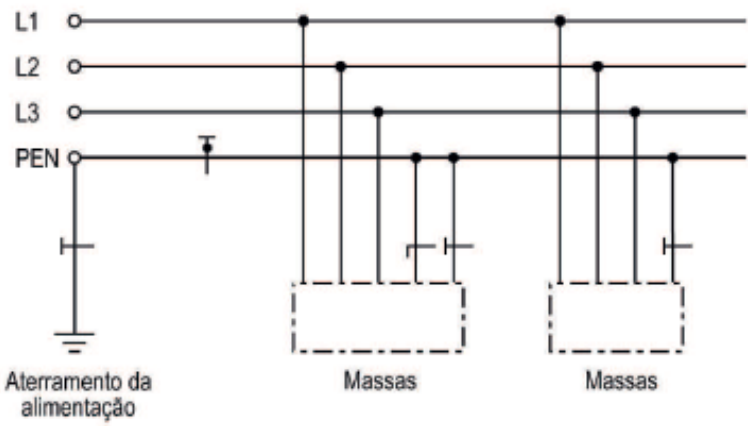

Figura 2: Representação do esquema TN-C.

Fonte: ABNT NBR 5410 (2004, p.16)

Esquema TN-C-S: possui um único condutor com as funções de neutro e proteção combinadas, em parte do esquema. Esquema representado na figura $3[1]$.

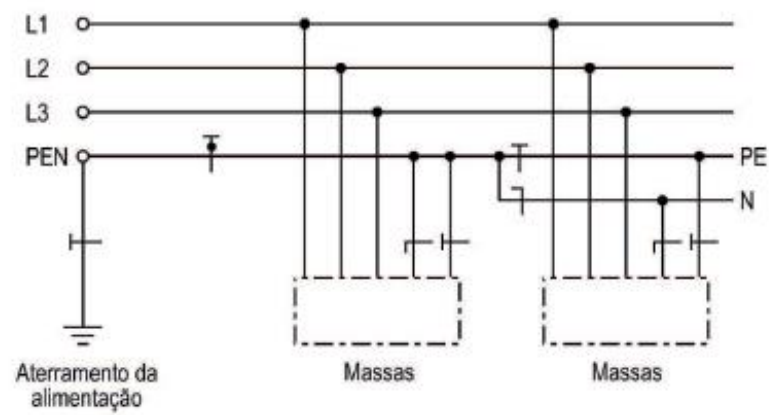

Figura 3: Representação do esquema TN-C-S.

Fonte: ABNT NBR 5410 (2004, p.15)

\subsection{Esquema TT}

Neste tipo de esquema, possui um ponto de alimentação e massa da instalação diretamente aterrados a eletrodos de aterramentos distintos. Esquema representado na figuras 4[1].

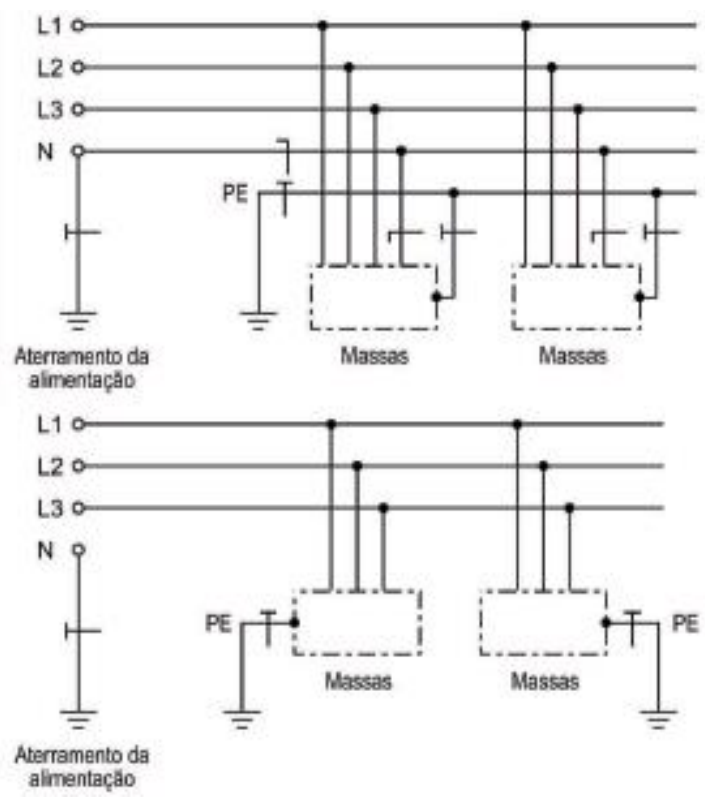

Figura 4: Representação do esquema TT. Fonte: ABNT NBR 5410 (2004, p.16)

\subsection{Esquema IT}

Neste tipo de esquema, possui as partes vivas isoladas da terra ou por um ponto de alimentação aterrado através de impedância, as massas da alimentação são aterradas. Esquema representado na figura $5[1]$. 


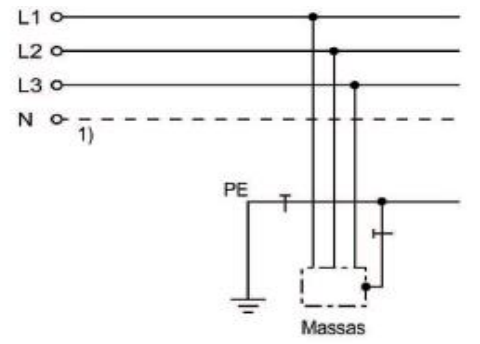

A
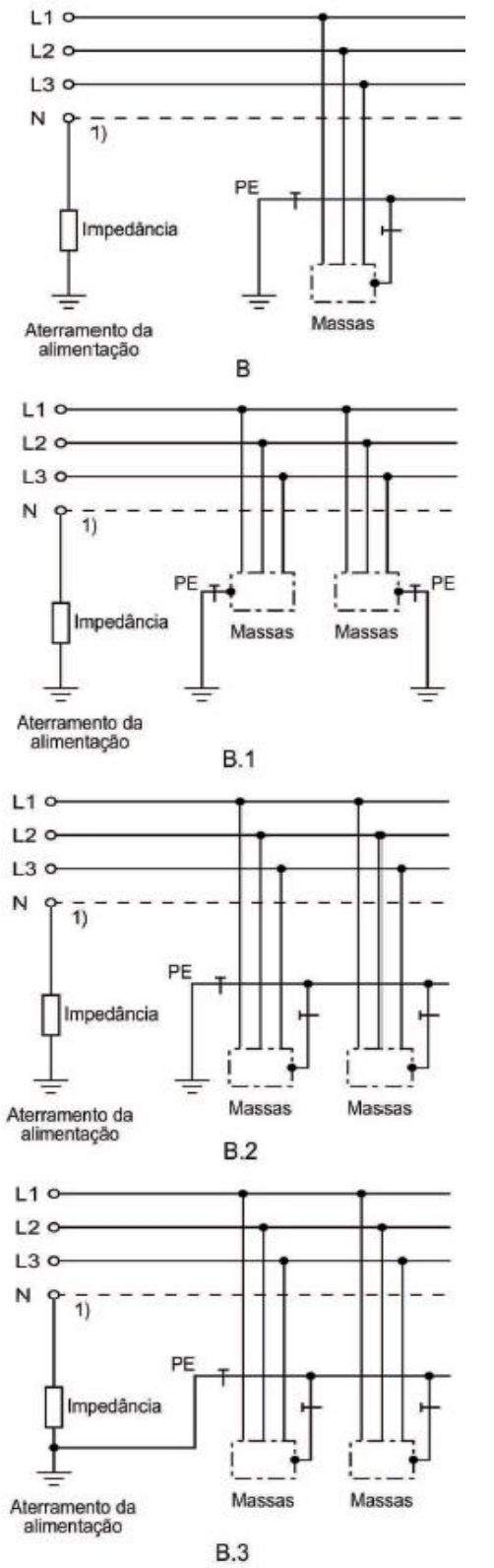

Figura 5: Representação do esquema TN-C-S. Fonte: ABNT NBR 5410 (2004, p.17)

A - Ausência de aterramento da alimentação.

B - Utilização de impedância no aterramento da alimentação;
B.1 - Presença de eletrodos de aterramento separados e independentes do

eletrodo de aterramento da alimentação para cada uma das massas;

B.2 - Presença de eletrodo incomum no aterramento das massas, porém,

separados do eletrodo de aterramento da alimentação.

B.3 - Presença de eletrodo de aterramento incomum entre alimentação das massas do sistema.

\section{Aterramento de um Sistema Fotovoltaico Micro e Minigeração Distribuida (Corrente Contínua)}

Até que a "NBR 16690: Instalações elétricas de arranjos fotovoltaicos - Requisito de projetos" seja publicada, adota-se a "NBR 5410 Instalações elétricas de baixa tensão", em que pode encontrar uma regulamentação de instalações de corrente contínua até 1500V, havendo também a "NBR5419 Proteção de estruturas contra descargas atmosféricas" para regulamentar a necessidade de aterrar as partes condutoras expostas do arranjos fotovoltaicos (conjunto de módulos fotovoltaicos), para fins de proteção contra surtos provocados por descargas atmosféricas.

Existem quatro opções para aterramento ou equipotencialização de arranjos fotovoltaicos[3]:

- Aterramento funcional das peças metálicas não energizadas.

- Aterramento de proteção contra descargas atmosféricas.

- Barramento de equipotencialização, com a função de evitar potenciais elétricos diferentes dentro de uma mesma instalação.

- Aterramento funcional do gerador fotovoltaico, aonde se aterra um polo do arranjo fotovoltaico.

A equipotencialização e aterramento das partes condutoras expostas devem ser realizados de acordo com a figura a seguir. 


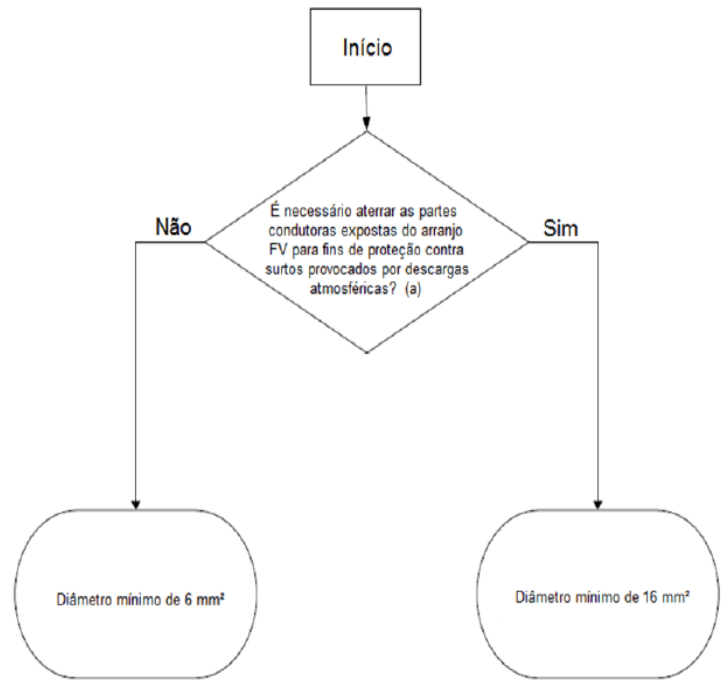

Figura 6: Fluxograma para escolha de condutores de aterramento e equipotencialização das partes condutoras expostas de arranjos fotovoltaicos.

Fonte: Projeto ABNT NBR 16690 (2016, p.55)

Ao analisar o fluxograma presente na Figura 6, pode-se determinar que a seção mínima de um condutor para aterrar as partes metálicas expostas é de $6 \mathrm{~mm}^{2}$ de cobre. Caso o sistema fotovoltaico tenha a necessidade de aterrar as suas partes condutoras expostas para fim de proteção contra descargas atmosféricas adota-se uma seção mínima de $16 \mathrm{~mm}^{2}$. Para que possa responder à pergunta da Figura 6, deve-se consultar a norma NBR 5419[3].

Tratando do lado de corrente contínua, é essencial que exista um barramento de equipotencialização que liga o inversor ao gerador fotovoltaico, para que protejam de sobretensões causadas por descargas atmosféricas. De acordo com o projeto de norma, o condutor do barramento de equipotencialização deve ser executado fisicamente o mais próximo possível dos condutores energizados, reduzindo, desta forma, laços nos condutores[3].

Quando se trata de aterramento funcional no lado de corrente contínua, está diretamente atrelado ao tipo de tecnologia utilizado no módulo e no inversor. Módulos Fotovoltaicos com tecnologia de filme fino devem ter um de seus condutores principais, positivo ou negativo, aterrados. Se recomenda, assim, que a ligação deste tipo de aterramento seja feita através de um resistor. O valor do resistor deve ser o mais alto valor de resistência admissível, conforme indicadas pelo fabricante. Já quando se trata de inversores, comumente não se pode aterrar os que não possuem transformador. [4]
Segundo o projeto de norma, todos os arranjos fotovoltaicos que possuírem aterramento funcional, a ligação do aterramento deve ser feita em um único ponto e este ser ligado ao terminal de aterramento da instalação elétrica. Normalmente existe uma grande distância entre os equipamentos do sistema fotovoltaico, desta forma sendo necessário a utilização de sistemas de aterramento distintos. Quando isto ocorrer, deve-se interconecta-los[3].

No lado de corrente alternada ocorre o aterramento funcional do sistema, em que a conexão entre o circuito elétrico e a terra é feito através do condutor de neutro, utilizando, deste modo, o aterramento do tipo TN em suas variações.

\section{Determinação da seção do condutor de proteção}

Segundo a norma NBR5410 existem duas maneiras de dimensionar o condutor de proteção. A primeira seria através do cálculo de seção mínima do condutor, que não deve ser menor que o obtido pela expressão[1]:

$$
S=\frac{\sqrt{I^{2} \times T}}{K}
$$

Sabe-se que:

$\mathrm{S}$ - seção mínima do condutor $\left(\mathrm{mm}^{2}\right)$;

I - corrente de falta (A);

T - tempo de atuação do dispositivo de proteção (s);

$\mathrm{K}$ - fator que depende do material do condutor de proteção, de sua isolação e outras partes, e das temperaturas inicial e final do condutor.

O segundo método para dimensionar a seção do condutor de proteção é determinada através da tabela1.

Tabela 1: Seção mínima do condutor de proteção

\begin{tabular}{|c|c|}
\hline $\begin{array}{c}\text { Seção dos condutores } \\
\text { de fase } \mathbf{S}\left(\mathbf{m m}^{2}\right)\end{array}$ & $\begin{array}{c}\text { Seção mínima do } \\
\text { condutor de proteção } \\
\text { correspondente }\left(\mathbf{m m}^{\mathbf{2}}\right)\end{array}$ \\
\hline $\mathrm{S} \leq 16$ & $\mathrm{~S}$ \\
\hline $16<\mathrm{S} \leq 35$ & 16 \\
\hline $\mathrm{S}>35$ & $\mathrm{~S} / 2$ \\
\hline
\end{tabular}

Fonte: ABNT NBR 5410 (2004, p.150) 
Para condutores de seção não padronizadas devese escolher o condutor que tenha seção mais próxima. Esta tabela só é válida para condutores que possuam mesmo material condutor que os condutores de fase. Vale salientar que um condutor de proteção pode ter dois ou mais circuitos em comum. Para que possa realizar a instalação desta forma deve-se instalar os condutores no mesmo conduto e calcular sua seção de acordo com um os dois métodos citados anteriormente[1].

\section{Dispositivo de Proteção contra surtos (DPS)}

O Dispositivo de proteção contra surtos, conhecido como DPS, tem a finalidade de proteger equipamentos eletrônicos conta surtos de tensão. Agem de modo a limitar sobretensões transitórias e desviam as correntes de surto para o sistema de aterramento.

Para proteger os equipamentos o DPS deve apresentar uma alta impedância quando não acionado. Assim que o dispositivo identifica um surto de tensão, sua impedância diminui de forma que permita drenar a correntes de surto para o sistema de aterramento.

Conforme a NBR5410 os DPS são divididos em três Classes[1]:

\section{Classe I:}

Destinado a proteger os equipamentos contra sobretensões provocadas por descargas atmosféricas. Recomenda-se serem instalados em quadros de entrada.

Classe II:

Destinado a proteger os equipamentos contra sobretensões provocadas por descargas indiretas e sobretensões de manobras. Recomenda-se serem instalados em quadros de distribuição.

\section{Classe III:}

Exercem um papel de terminação, impondo uma baixa tensão residual. Recomenda-se serem instalados próximos aos aparelhos eletrônicos que deseja proteger.

Em sistemas fotovoltaicos deve-se utilizar este tipo de dispositivo para proteger principalmente inversores e módulos fotovoltaicos. A realização da análise de risco seguindo a metodologia da NBR5419 é que vai definir se existe a real necessidade de utilização do DPS.

Baseado na norma IEC 61643, devemos instalar os DPS com níveis de proteções inferiores aos suportados pelos equipamentos. A tabela a seguir mostra valores de tensões impulsivas que os equipamentos que compões um sistema de geração fotovoltaico suporta[8].

Tabela 2: Suportabilidade a tensões impulsivas de equipamentos que compõem o sistema de geração fotovoltaica.

\begin{tabular}{|c|c|c|c|c|}
\hline \multicolumn{5}{|c|}{$\begin{array}{c}\text { Suportabilidade a tensões impulsivas de } \\
\text { equipamentos que compõem o sistema de } \\
\text { geração fotovoltaica }\end{array}$} \\
\hline \multirow{2}{*}{$\begin{array}{c}\text { Uocmax } \\
\text { (V) }\end{array}$} & $\begin{array}{c}\text { Suportabilidade a tensões impulsivas } \\
\text { - Uw(KV) }\end{array}$ \\
\cline { 2 - 2 } & $\begin{array}{c}\text { Mod. } \\
\text { Classe } \\
\text { B }\end{array}$ & \multirow{4}{*}{ Inversor } & $\begin{array}{c}\text { Outros } \\
\text { equipa- } \\
\text { mentos }\end{array}$ & $\begin{array}{c}\text { Mod. } \\
\text { Classe } \\
\text { A }\end{array}$ \\
\hline 100 & 0,8 & & 0,8 & 1,5 \\
\hline 150 & 1,5 & \multirow{2}{*}{2,5} & 1,5 & 2,5 \\
\hline 300 & 2,5 & & 2,5 & 4 \\
\hline 424 & 4 & & 4 & 4 \\
\hline 600 & 4 & & 4 & 6 \\
\hline 800 & 5 & 4 & 5 & 6 \\
\hline 849 & 6 & & 6 & 8 \\
\hline 1000 & 6 & 6 & 6 & 8 \\
\hline 1500 & 8 & 8 & 8 & 12 \\
\hline
\end{tabular}

Fonte: Proteção De Equipamentos Elétricos E Eletrônicos Contra Surtos Elétricos Em Instalações (2016, p.220)

\section{Proteção contra sobrecorrentes}

Em sistemas fotovoltaicos, os módulos solares se comportam como uma fonte de corrente contínua, cuja corrente varia de acordo com a irradiância e a temperatura[7].

Como as correntes de curto-circuito e as correntes de máxima potência têm valores próximos, todos os dispositivos desses tipos de sistemas devem ser dimensionados pela corrente de curto circuito[7].

Sabe-se que para se colocar séries fotovoltaicas em paralelo, tem que garantir que estejam com mesma tensão, pois ao colocar fontes como diferentes tensões em paralelo a fonte com menor tensão irá receber uma injeção de corrente reversa, pois irá se comportar como uma carga. Desta forma, verifica-se 
a necessidade de uma proteção contra corrente reversa através de Disjuntores ou Chaves fusíveis [7].

Deve-se proteger as séries fotovoltaicas sempre que existir possibilidade de corrente reversa maior que a suportada pelo módulo fotovoltaico. Podemos determinar esta necessidade pela seguinte expressão[7]:

$(\mathrm{SA}-1) \times \mathrm{Isc}>\mathrm{IR}$

Sabe-se que:

SA - Número de séries em paralelo;

Isc - Corrente de curto-circuito do módulo em condições STC;

IR - Máxima corrente reversa que o módulo suporta.

Em séries fotovoltaicas deve-se instalar o dispositivo de proteção no ponto que os condutores se reúnem formando um subarranjo ou arranjo fotovoltaico. O seu dimensionamento deve ser realizado pela seguinte expressão[7]:

$$
\begin{aligned}
& (1,5 \times \text { Isc })<\text { IP }<(2,4 * \text { Isc }) \\
& \text { IP } \leq \text { IR }
\end{aligned}
$$

Sabe-se que:

Isc - Corrente de curto-circuito do módulo em condições STC;

IP - Corrente de atuação do dispositivo de proteção;

IR - Máxima corrente reversa que o módulo suporta.

Em subarranjos fotovoltaicos deve-se instalar o dispositivo de proteção no ponto que os condutores se reúnem formando o arranjo fotovoltaico. O seu dimensionamento deve ser realizado pela seguinte expressão[7]:

$$
(1,25 \times \text { Isc sarj })<\mathrm{IP}<(2,4 * \text { Isc sarj })
$$

Sabe-se que:

Isc sarj - Corrente de curto-circuito do subarranjo em condições STC;

IP - Corrente de atuação do dispositivo de proteção;

Em arranjos fotovoltaicos deve-se instalar o dispositivo de proteção no ponto que os condutores se conectam ao Inversor. O seu dimensionamento deve ser realizado pela seguinte expressão[7]:

$$
(1,25 \times \text { Isc arj })<\mathrm{IP}<(2,4 * \text { Isc arj })
$$

Sabe-se que:

Isc arj - Corrente de curto-circuito do arranjo em condições STC;

IP - Corrente de atuação do dispositivo de proteção;

\section{Conclusões}

Em sistemas fotovoltaicos conectados à rede de micro e minigeração é necessário fazer o aterramento de proteção dos equipamentos, ou seja, conectar a carcaça de partes condutoras expostas ao sistema de terra. Já o aterramento funcional do sistema normalmente acontece na conexão do circuito elétrico ao sistema de aterramento, através do condutor neutro no lado de corrente alternada[3].

$\mathrm{O}$ aterramento funcional no lado de corrente contínua, depende da tecnologia de módulos ou de inversores utilizadas. Em geral, deve-se sempre verificar os procedimentos recomendados pelo fabricante no manual dos equipamentos a serem instalados[3].

Os sistemas fotovoltaicos estão sujeitos ao efeito de sobretensões nas instalações DC do gerador, para realizar a proteção dos equipamentos é muito importante que se instalem DPS que estejam de acordo com a norma IEC 61643.

Como visto, não é comum sobrecorrentes nas instalações de corrente contínua de sistemas fotovoltaicos, pois os painéis solares são fontes limitadas de corrente. Porém a norma determina o uso de proteção para correntes reversas no módulo[7].

\section{Referências}

[1] ABNT NBR 5410-2004 - Instalações elétricas de baixa tensão; da Associação Brasileira de Normas Técnicas (ABNT).

[2] ABNT NBR 5419- 2015 - Proteção de estruturas contra descargas atmosféricas; Associação Brasileira de Normas Técnicas (ABNT).

[3] Projeto ABNT NBR 16690 - 2016 - Instalações elétricas de arranjos fotovoltaicos - Requisitos de projeto; Associação Brasileira de Normas Técnicas 
(ABNT).

[4] PINHO, João Tavares. GALDINO, Marco Antônio (organizadores). Manual De Engenharia Para Sistemas Fotovoltaicos. Rio de Janeiro:2004. Disponível em:http://www.cresesb.cepel.br/publicacoes/do wnload/Manual_de_Engenharia_FV_2014.pdf. Acessado em 09/01/2019.

[5] PEREIRA, Filipe. Guia De Manutenção De Instalações Fotovoltaicas. Lisboa: Publindústria, $1^{\text {a Ed., }} 2012$.

[6] NASCIMENTO, Douglas Aguiar; IANO, Yuzo; LOSCHI, Hermes José; DE CARVALHO, Silvio Renato Messias. Instalações De Sistemas De Geração Solar Fotovoitaca. Disponível em:https://www.researchgate.net/profile/Herme s_Jose_Loschi/publication/311452392_Instalacoe s_de_Sistemas_de_Geracao_Solar_Fotovoltaica_ Um_estudo_sobre_sistemas_de_aterramento_pr otecao_contra_surto_e_descargas_atmosfericas/I inks/5846fc2308ae2d217570382f/Instalacoesde-Sistemas-de-Geracao-Solar-Fotovoltaica-Umestudo-sobre-sistemas-de-aterramento-protecaocontra-surto-e-descargas-atmosfericas.pdf.

Acessado em 15/01/2019.

[7] AYRÃO,Vinicius. Energia Solar Fotovoltaica No Brasil Conceitos, Aplicações E Estudo De Caso. Rio de Janeiro:2018. Disponível em: https:/ /www.leonardoenergy.org.br/wpcontent/uploads/2019/05/Estudo s-Fotovoltaicos-Vinicius-Ayrao.pdf.Acessado em 20/05/2019

[8] PAULINO, José Osvaldo Saldanha; BARBOSA, Célio Fonseca; MOREIRA; Ronaldo Kascher; BARBOSA, Wagner Almeida; LOBO, Marcelo Augusto Freire; LOBO, Ailton Ricaldoni. Proteção De Equipamentos Elétricos E Eletrônicos Contra Surtos Elétricos Em Instalações. Lagoa Santa:2016, Editora Campler, 1a Ed., 2016. 